\title{
A behavioral field approach to operant conditioning: Extinction-induced sanddigging
}

\author{
PAUL T. P. WONG \\ Trent University, Peterborough, Ontario, Canada K9J $7 B 8$
}

\begin{abstract}
In a free-operant situation, food reinforcement of panel-pushing decreased alternative responses, while extinction increased the complexity and variability of behavioral fields. In most subjects, extinction-induced sanddigging first increased and then returned to baseline. The present extinction data support a stage model of extinction (Wong, 1978).
\end{abstract}

The behavioral field approach to learning as employed by Wong $(1977,1978)$ has demonstrated that reinforcement and extinction operations affect not only the criterion response, but other aspects of behavior as well. Different from a multiple-response baseline analysis (e.g., Dunham, 1971), the behavioral field approach also investigates sequential structures and other properties that are characteristic of behavior as a whole rather than its individual components (Wong, in press). On the basis of a behavioral field analysis of discrete-trial runways data, a stage model of extinction has been formulated (Wong, 1978). Briefly, the model posits that in the first habit stage, the animal perseverates on the old habit that has been reinforced; in the second trial-and-error stage, the animal explores various response elements in its response repertoire and different stimulus elements in its environment; in the third resolution stage, the animal either settles for an alternative goal, or, in the absence of such a goal, becomes "helpless" and "depressed." Evidence for extinction-induced "helplessness" has been obtained (Wong, Note 1).

The present study employed the behavioral field approach to test the stage model in a free-operant situation, where several responses (e.g., sanddigging and drinking) were measured during baseline sessions as well as during reinforcement and extinction of a panel-pushing response. Since spontaneous sanddigging is affected by food deprivation and is considered to be related to food seeking (Wong, in press), it was predicted that sanddigging should be higher than baseline only during the middle stage of extinction. It was also predicted that the animals should exhibit greater complexity and variability in behavioral fields during the middle stage of extinction. With prolonged extinction, all behaviors should return to baseline levels. Inasmuch as spontaneous sanddigging may function as an alternative goal during Stage 3

This work was supported by National Research Council Grants $3 \mathrm{~A} 8635$ and $3 \mathrm{A0701}$. The author thanks P. Moore, N. White, and W. Grife for their assistance and A. F. Carr for helpful comments on an earlier draft. Requests for reprints should be addressed to P. T. P. Wong, Department of Psychology, Trent University, Peterborough, Ontario, Canada K9J 7 B8. extinction (Wong, 1978), "depression" in the form of passivity or inactivity was not predicted.

\section{METHOD}

\section{Subjects}

The subjects were four male and four female light-hooded rats of Walker-Walker stock, bred in the vivariam of Trent University. They were approximately 100 days old and maintained at $80 \%$ of free-feeding body weights. Water was always available in individual cages.

\section{Apparatus}

The testing chamber was the same as described by Wong (in press). Briefly, it contained six manipulanda: a food-cup panel for pushing, a bar for pressing, a pair of blades for biting, a chain for pulling, sand for digging, and a water spout for drinking. These manipulanda, together with a manual keyboard, were connected to an Esterline Angus chart recorder. Any response on the manipulanda was automatically recorded on the chart, which moved at the speed of $15.24 \mathrm{~cm} / \mathrm{min}$. The manual keyboard provided measures of observed behaviors (i.e., grooming, exploration, and immobility).

\section{Procedure}

In all phases, subjects were placed in the testing chamber, one at a time, for $10 \mathrm{~min} /$ day. Phase 1 consisted of 16 days of baseline measures. In Phase 2, each food-cup panel push was reinforced by a 45 -mg pellet. Since males showed a slower rate of acquisition, they were given 5 days of acquisition training, while females were given 4 days of acquisition. In Phase 3 , all subjects were given 12 days of extinction training, during which food-cup panel response was no longer reinforced by food pellets or food-magazine clicks.

\section{RESULTS}

Data of individual subjects are shown in Figure 1. In Phase 1, data of the last four sessions only are presented, because most behaviors were stabilized during these sessions. Biting response occurred so rarely that it was not included in the graph. In terms of response duration, the following findings were quite apparent from inspection of the graph: (1) Reinforcement of food-cup panel push increased this response and decreased alternative responses. (2) Extinction resulted in a rapid decrease in the food-cup response, and an increase in alternative responses. (3) Sanddigging, the dominant 


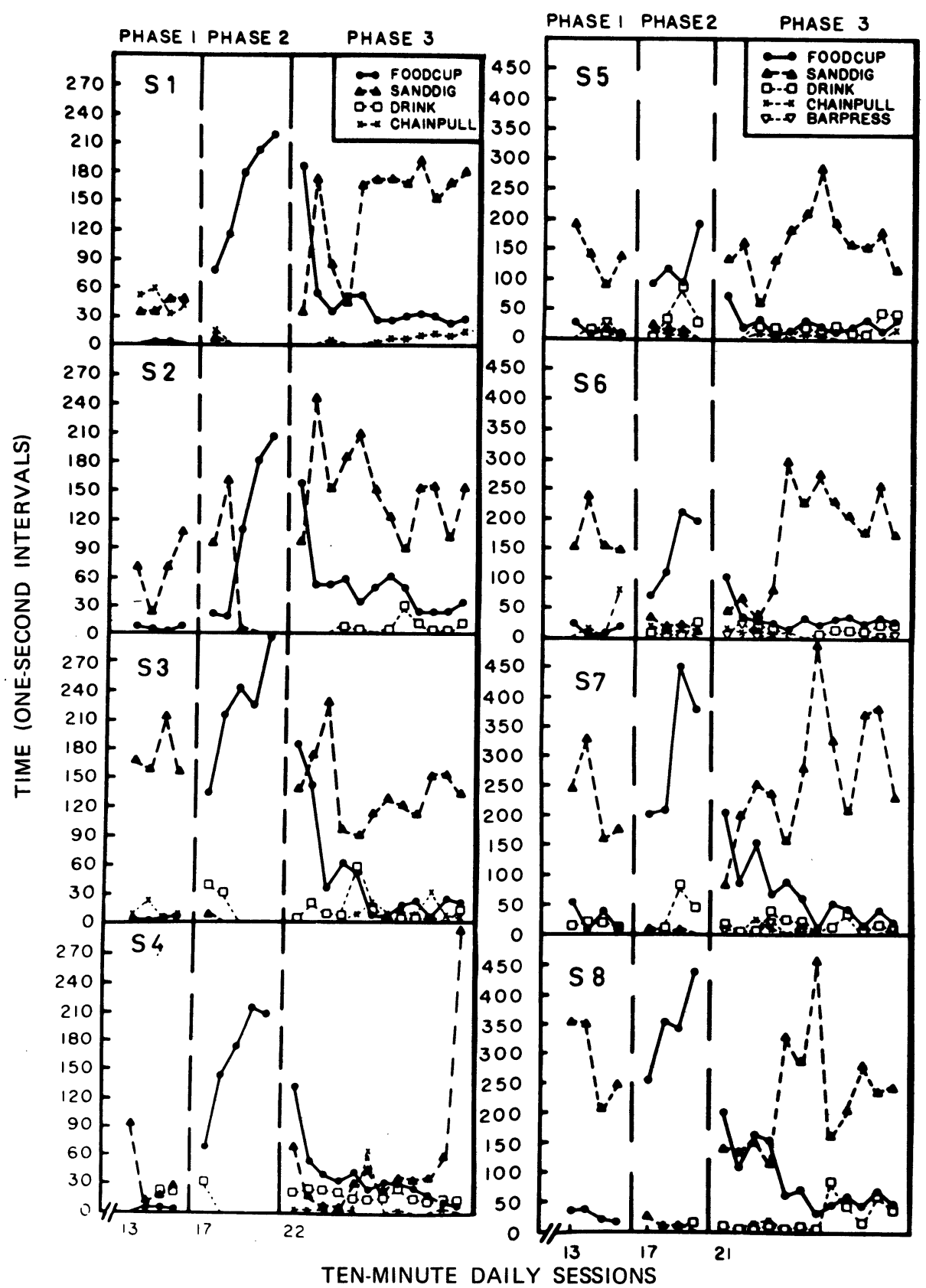

Figure 1. Data of individual subjects. The left panel consists of males; the right panel, females. Note that females had a different scale due to a higher level of sanddigging.

competing response, exceeded its baseline during extinction in all subjects. (4) Except for S1 and S4, sanddigging showed the tendency of returning to baseline.

Extinction was divided into three blocks of four sessions per block to represent the first, second, and last stages of extinction. Granted that there are individual differences in the amount of extinction training needed to reach the last stage, such an arbitrary division nevertheless does provide a fairly good approximation and permits statistical analysis. Results of analysis of variance confirmed the prediction that sanddigging in Block 2 was significantly higher than baseline (i.e., the last four sessions) $[F(1,7)=5.79, p<.05]$. Neither Block 1 nor Block 3 differed significantly from baseline. Analysis of the three extinction blocks revealed a significant main effect of blocks $[F(2,14)=5.19, \mathrm{p}<.05]$; however, the effect was mainly due to the difference between Block 2 and the other two blocks of extinction $[F(1,14)=4.94, p<.05]$. 
As sanddigging increased, grooming, exploration, and immobility decreased somewhat, but there were no significant changes in these responses in the course of extinction as well as in comparison with baseline. Recent research has shown that most rats show an increase in immobility response after four or five sessions of extinction, when there is no opportunity for sanddigging (Wong, Note 1). In the present study, immobility response actually showed a slight decreasing trend during extinction.

Complexity of the behavioral field was measured by number of different responses (i.e., exploration, grooming, immobility, and the six different kinds of manipulanda). Mean numbers of different responses for baseline and the three extinction blocks $(1,2$, and 3$)$ were $5.62,6.53,6.69$, and 6.69 , respectively. In all three extinction blocks, complexity was greater than in baseline $[\mathrm{Fs}(1,7)=8.77,182.91$, and 106.47 , ps $<.05$, .001 , and .001, respectively]. Variability of the behavioral field was measured by different behavioral transitions. For example, exploration to sanddigging and sanddigging to exploration represent two different behavioral transitions. Mean numbers of different transitions for baseline and the three extinction blocks were $11.78,15.25,15.03$, and 15.37 , respectively. In all three extinction blocks; variability was greater than in baseline $[\mathrm{Fs}(1,7)=24.68,23.59$, and 18.37, respectively, all ps $<.01]$.

\section{DISCUSSION}

Similar to punishment (Dunham, 1971), extinction suppressed the criterion response and increased alternative responses. The increase in the unpunished alternatives has been attributed to implicit avoidance or interaction of competing responses (Dunham, 1971, 1972). According to the first interpretation, the increase is assumed to be motivated by an implicit avoidance contingency, because the longer a subject spends in an unpunished alternative, the longer it is "safe" from punishment. According to the interaction hypothesis, each response in the animal's response repertoire occupies a certain portion of time available to it, and time spent in one response necessarily reduces time available to an incompatible response. In other words, there is an interaction or reciprocal inhibition between competing responses in time and space.

Both of these interpretations are inadequate for the present data. If an increase in sanddigging was reinforced by an implicit avoidance contingency, then it should show a monotonic increasing function rather than an inverted-U function, as was observed in all subjects, except S1 and S4. The interaction hypothesis also cannot account for the inverted-U function of sanddigging. Further, neither hypothesis permits one to predict which alternative response would predominate when the criterion response is no longer reinforced. A change in response hierarchy from baseline to extinction poses a special problem for the interaction hypothesis. For example, in S1 the dominant response was exploration during baseline, but sanddigging became dominant during extinction. In fact, in all subjects sanddigging surpassed baseline, while exploration, grooming, and immobility fell slightly below baseline. Such change from baseline to extinction cannot be simply attributed to a decrease in food-cup response time, as suggested by the interaction hypothesis.

As well, the complexity and variability data were not readily interpretable by either the implicit avoidance hypothesis or the interaction hypothesis. According to the former, the dominant response should become more dominant and the behavioral field should become less complex and less variable during extinction because of the implicit avoidance (i.e., negative reinforcement) contingency. According to the latter, both complexity and variability measures should be the same during extinction as during baseline. Staddon and Simmelhag (1971) have stated that "training involves a progressive reduction in variability under the selection action of reinforcement... so the absence of reinforcement (extinction) represents a relaxation of selection" (p. 24). However, the present data show that extinction involves more than the passive relaxation of selection, because both complexity and variability measures exceeded baseline.

The present findings implicate an additional source of motivation not present during baseline. This additional source is hypothesized to be frustrative nonreward. According to Wong's (1978) stage model of extinction, nonreward should initially invigorate the old habit (Stage 1) and then increase the amount, scope, and variability of alternative responses (Stage 2); finally, the animal should either settle for some kind of alternative activities or become depressed (Stage 3). In the present study, invigorated responses directed to the food cup (e.g., a high rate of panel pushing, vigorous digging into the food cup) were observed mainly in the first two extinction sessions, reflecting Stage 1 extinction. The curvilinear function of sanddigging provides evidence of Stage 2 extinction. It should be noted that in free-operant situations, there are stage transitions both within extinction sessions and between sessions. Even in the first extinction session, after a period of invigorated food-cup response, most rats began to explore other alternatives toward the end of the first session. In subsequent sessions, the tendency to switch to alternative behaviors increased and became dominant. One reason why complexity and variability measures were less sensitive to between-sessions stage transitions than was sanddigging might be due to different scales of measurement. While complexity and variability were based on the number of predetermined categories of behavior involved per session, sanddigging was based on time spent in a particular category of behavior.

Sanddigging, as well as other alternative behaviors, showed a trend toward baseline during the last few sessions in all subjects except S1 and S4. According to the stage model of extinction, such a tendency reflects the eventual subsiding of frustration. However, Wong (Note 1) has found that when opportunities for sanddigging and other alternative activities were not provided, frustration eventually gave way to "depression," as indicated by a high degree of passivity as well as deficits in learning a new task.

The present behavioral field approach to studying extinction in a free-operant situation has shown that extinction may be different from punishment in that, while both operations are effective in reducing the strength of the criterion response, only extinction has the side effect of increasing the amount, scope, and variability of alternative responses.

\section{REFERENCE NOTE}

1. Wong, P. T. P. Extinction-induced facilitation and "learned helplessness." Paper presented in a symposium on "Learned helplessness and frustration" in the XIXth International Congress of Applied Psychology, Munich, Germany, August 1978.

\section{REFERENCES}

Dunham, P. J. Punishment: Method and theory. Psychological Review, 1971, 78, 58-70. 
Dunham, P. J. Some effects of punishment upon unpunished responding. Journal of the Experimental Analysis of Behavior, 1972, 17, 443-450.

Staddon, J. E. R., \& Simmelhag, V. L. The "superstition" experiment: A reexamination of its implication for the principles of adaptive behavior. Psychological Review, 1971, 78, 3-43.

Wong, P. T. P. A behavioral field approach to instrumental learning: I. Partial reinforcement effects and sex differences. Animal Learning \& Behavior, 1977, 5, 5-13.
Wong, P. T. P. A behavioral field approach to instrumental learning in the rat: II. Training parameters and a stage model of extinction. Animal Learning \& Behavior, 1978, 6. 82-93.

Wong, P. T. P. A behavioral field approach to general activity: Sex differences and food deprivation in the rat. Animal Learning \& Behavior, in press.

(Received for publication June 6, 1978.) 